\title{
Momentum-dependent mean field based upon the Dirac-Brueckner approach for nuclear matter
}

\author{
G. Q. Li and R. Machleidt \\ Department of Physics, University of Idaho, Moscow, ID 83843, USA
}

\begin{abstract}
A momentum-dependent mean field potential, suitable for application in the transport-model description of nucleus-nucleus collisions, is derived in a microscopic way. The derivation is based upon the Bonn meson-exchange model for the nucleon-nucleon interaction and the Dirac-Brueckner approach for nuclear matter. The properties of the microscopic mean field are examined and compared with phenomenological parametrizations which are commonly used in transport-model calculations.
\end{abstract}

Typeset using REVTEX 
The microscopic description of nuclear matter, finite nuclei, and nuclear reactions in terms of the realistic nucleon-nucleon $(\mathrm{NN})$ interaction continues to be an interesting topic in nuclear physics. Although quantum chromodynamics (QCD) is believed to be the ultimate theory of strong interaction, the only quantitative NN potential models available up till now are based on the idea of meson exchange; a well-known example is the Bonn potential [1,2].

Special many-body theories, such as the Brueckner approach and the variational method, have been developed such that a realistic NN interaction can be applied in nuclear manybody calculations. Nuclear matter saturation has been quantitatively explained by the DiracBrueckner-Hartree-Fock (DBHF) approach [3 [6], starting from a realistic NN interaction. Thus, this approach provides a natural starting point for the self-consistent description of nuclear matter, nuclear structure, and nuclear reactions in terms of the realistic NN interaction.

The extension of the DBHF approach from nuclear matter to the structure of finite nuclei and nucleon-nucleus scattering have been attempted [7 [1]. The direct solution of the DBHF equation in finite nuclei is, however, rather involved. More practically, one either parametrizes the DBHF results in nuclear matter in terms of simple Lagrangians [9 12] that can be easily applied in finite nuclei, or one performes a full Brueckner-Hartree-Fock (BHF) calculation in the finite nucleus taking the relativistic effects via the local-density approximation into account [8].

In tune with the latter approach, we present in this paper a momentum-dependent mean field single-particle potential derived from DBHF nuclear matter calculations. This mean field potential is suitable for application in the transport-model description of nucleusnucleus collisions. Together with the in-medium NN cross sections derived in our earlier work [13], a fully self-consistent calculation of nucleus-nucleus collisions can then be performed.

A self-consistent approach has been pursued in Refs. [14 17] applying the Reid softcore potential [18] in a non-relativistic Brueckner-Hartree-Fock (BHF) calculation. The major criticism [19] of this study comes from the fact that the BHF calculation cannot even reproduce quantitatively the saturation properties of static nuclear matter [20]; the 
application of its results to colliding nuclear matter is thus debatable.

From the theoretical point of view, nucleus-nucleus collisions at intermediate energies are of particular interest. At these energies, both the mean field and two-body collisions play an equally important role in the dynamical evolution of the two colliding systems; therefore, they have to be taken into accout on an equal footing in transport models such as the Boltzmann-Uehling-Uhlenbeck (BUU) equation [19] or quantum molecular dynamics (QMD) [21].

In the early applications of transport models to nucleus-nucleus collisions, the mean field and the in-medium NN cross sections were parametrized separately: a simple Skyrme-type parametrization was used for the mean field [19], while for the in-medium NN cross sections free-space proton-proton $(p p)$ data were applied [22,19]. By varying the mean field (i.e., the parameters in the Skyrme parametrization) — which is related to the nuclear equation of state - and by comparing theoretical results with experimental data, one expects to obtain some information on the nuclear equation of state.

There are at least two uncertainties in this approach: the mean field and the in-medium NN collisions. Some observables that are believed to carry useful information about the nuclear equation of state are also strongly affected by what is assumed for the NN collisions. Therefore, the in-medium NN cross sections used in transport models should be determined consistently before reliable information concerning the equation of state can be obtained. Within the DBHF approach, the in-medium NN cross sections below pion threshold have been studied in Ref. [13]. Using those microscopic in-medium NN cross sections will reduce the uncertainty concerning the in-medium NN collisions.

Furthermore, it is well known that - due to correlations and exchange terms - the effective nuclear interaction is momentum dependent. Empirically, the momentum dependence of the mean field is best illustrated by the nucleon effective mass $m^{*} / m$ which is about 0.7 at low energies and approaches unity at high energies [23]. Momentum dependence of the mean field has been found to play an important role in observables that are claimed to carry useful information about the nuclear equation of state, such as transverse momentum 
transfer [24,25] and particle production [26] in nucleus-nucleus collsions. The microscopically derived momentum dependence of the mean field may help reducing also this source of uncertainty.

Our derivation of the mean-field potential is based upon the DBHF approach for nuclear matter. For a comprehensive and pedagogical introduction into the basic ideas of the DiracBrueckner approach and its formalism, we refer the interested reader to section 10.5 of Ref. [2] and Ref. [6]. Here, we will only briefly summarize the major points and the basic equations. For the NN potential, we will apply the one-boson-exchange representation of the Bonn meson-exchange model for the NN interaction [27]. It includes six nonstrange mesons with given mass and coupling. Pseudovector (derivative) coupling is used for pseudoscalar mesons $(\pi$ and $\eta)$. A form factor of monopole type is applied at each meson-nucleon vertex which simulates the short-range dynamics of quark-gluon nature. The potential provides an accurate description of the deuteron and NN scattering [2,6].

The essential point of the DBHF approach is the use of the Dirac equation for the description of single-particle motion in the nuclear medium

$$
\left[\boldsymbol{\alpha} \cdot \mathbf{p}+\beta\left(m+U_{S}\right)+U_{V}\right] \tilde{u}(\mathbf{p}, s)=\epsilon \tilde{u}(\mathbf{p}, s)
$$

where $U_{S}$ is an attractive scalar field and $U_{V}$ the time-like component of a vector field which is repulsive; $m$ denotes the experimental mass of the free nucleon.

The positive-energy Dirac spinors that solve Eq. (1) can be written as

$$
\tilde{u}(\mathbf{p}, s)=\left(\frac{\tilde{E}_{\mathbf{p}}+\tilde{m}}{2 \tilde{m}}\right)^{\frac{1}{2}}\left(\begin{array}{c}
1 \\
\frac{\boldsymbol{\sigma} \cdot \mathbf{p}}{\tilde{E}_{\mathbf{p}}+\tilde{m}}
\end{array}\right) \chi_{s}
$$

with

$$
\begin{gathered}
\tilde{m}=m+U_{S}, \\
\tilde{E}_{\mathbf{p}}=\left(\tilde{m}^{2}+\mathbf{p}^{2}\right)^{\frac{1}{2}}
\end{gathered}
$$

and $\chi_{s}$ a Pauli spinor. The normalization is $\overline{\tilde{u}} u=1$. 
Similar to conventional Brueckner theory, the basic quantity in the DBHF approach is a $\tilde{G}$-matrix which satisfies the in-medium Thompson equation (relativistic Bethe-Goldstone equation) [2,6, 29]

$$
\begin{aligned}
\tilde{G}\left(\mathbf{q}^{\prime}, \mathbf{q} ; \mathbf{P}\right)= & \tilde{V}\left(\mathbf{q}^{\prime}, \mathbf{q}\right)+\mathcal{P} \int \frac{d^{3} k}{(2 \pi)^{3}} \tilde{V}\left(\mathbf{q}^{\prime}, \mathbf{k}\right) \frac{\tilde{m}^{2}}{\tilde{E}_{(1 / 2) \mathbf{P}+\mathbf{k}}^{2}} \\
& \times \frac{Q(\mathbf{k}, \mathbf{P})}{2 \tilde{E}_{(1 / 2) \mathbf{P}+\mathbf{q}}-2 \tilde{E}_{(1 / 2) \mathbf{P}+\mathbf{k}}} \tilde{G}(\mathbf{k}, \mathbf{q} ; \mathbf{P})
\end{aligned}
$$

For a pair of interacting nucleons with momentum $\mathbf{p}_{1}$ and $\mathbf{p}_{2}$ in the nuclear matter rest frame, the center-of-mass (c.m.) momentum is $\mathbf{P}=\mathbf{p}_{1}+\mathbf{p}_{2}$ and their relative momentum $\mathbf{q}=(1 / 2)\left(\mathbf{p}_{1}-\mathbf{p}_{2}\right) . \mathcal{P}$ denotes the principal value. Note that Eq. (5) is density dependent due to the Pauli projector, $Q$, and the scalar field, $U_{S}$; in the notation used in Eq. (5), this density dependence is suppressed. Notice also that $\tilde{V}$ is density dependent due to the inmedium Dirac spinors, Eq. (2), representing the four outer legs of the one-meson-exchange Feynman diagrams defining the potential. The in-medium Dirac spinors depend on $\tilde{m}$ which through $U_{S}$ depends on the density of the medium.

The Dirac equation and the in-medium Thompson equation are solved self-consistently. The nuclear matter properties are derived from the $\tilde{G}$-matrix. For more details, see section 10.5 of Ref. [2] and Ref. [6].

According to Eq (1), the single-particle energy of a nucleon with momentum $\mathbf{p}_{i}$ is

$$
\begin{aligned}
\epsilon_{i} & =\frac{\tilde{m}}{\tilde{E}_{i}}<i\left|\gamma \cdot \mathbf{p}_{i}+m\right| i>+\frac{\tilde{m}}{\tilde{E}_{i}} U_{S}+U_{V} \\
& =\frac{m \tilde{m}+\mathbf{p}_{i}^{2}}{\tilde{E}_{i}}+\frac{\tilde{m}}{\tilde{E}_{i}} U_{S}+U_{V} \\
& =\tilde{E}_{i}+U_{V}
\end{aligned}
$$

In our notation, $\mid i>$ is a single particle state represented by a Dirac spinor of the kind Eq. (2) and $<i \mid$ is the adjoint Dirac spinor, $\overline{\tilde{u}} \equiv \tilde{u}^{\dagger} \gamma_{o} ;\langle i| i>=1 ; \beta \boldsymbol{\gamma}=\boldsymbol{\alpha} ; \beta=\gamma_{0}$.

The scalar and vector field, $U_{S}$ and $U_{V}$, are determined from

$$
\frac{\tilde{m}}{\tilde{E}_{i}} U_{S}+U_{V}=\operatorname{Re} \sum_{j \leq k_{F}} \frac{\tilde{m}}{\tilde{E}_{i} \tilde{E}_{j}}<i j|\tilde{G}| i j-j i>
$$


This is the relativistic analogue to the non-relativistic Brueckner-Hartree-Fock definition of a single-particle potential using the effective mass approximation. Self-consistency of the DBHF calculations means satisfying Eq. (6).

As shown in Ref. [30], it is a good approximation to assume $U_{S}$ and $U_{V}$ to be momentum independent (note, however, that they are density dependent). Notice also that momentumindependence of $U_{S}$ and $U_{V}$ does not imply momentum-indepence of the mean field (cf. Eq. (13), below). Momentum-dependence of $U_{S}$ and $U_{V}$ is one possible source of momentumdependence of the mean field, but it is the less important one (see discussion below).

In order to define a mean-field potential, we rearrange the single-particle energy, Eq. (8),

$$
\epsilon_{i}=E_{i}+U_{V}+\tilde{E}_{i}-E_{i}=E_{i}+U_{i}
$$

with the free energy

$$
E_{i}=\left(\mathbf{p}_{i}^{2}+m^{2}\right)^{1 / 2}
$$

and the mean-field potential

$$
U_{i}=U_{V}+\tilde{E}_{i}-E_{i}
$$

More explicitly, this mean field is

$$
U(\rho, p)=U_{V}(\rho)+\left[\left(m+U_{S}(\rho)\right)^{2}+\mathbf{p}^{2}\right]^{1 / 2}-\left(m^{2}+\mathbf{p}^{2}\right)^{1 / 2}
$$

where the dependence on the density $\rho$ is now clearly indicated $(p \equiv|\mathbf{p}|)$. $U_{S}(\rho)$ and $U_{V}(\rho)$ are listed for many densities in Table VII of Ref. [6]; e. g., for $\rho_{0}=0.17 \mathrm{fm}^{-3}$ one has $U_{S}=-355.7 \mathrm{MeV}$ and $U_{V}=274.7 \mathrm{MeV}$. By using this table in a spline interpolation (adding $U_{S}=U_{V}=0$ for $\rho=0$ ), one can obtain $U_{S}$ and $U_{V}$ with good accuracy for any density between 0 and $4.2 \rho_{0}$. For a simple parametrization of our mean field potential, see Eq. (20), below.

The mean field, Eq. (13), shows strong momentum dependence for small $p$. For large $p(p>>m)$, the square roots are $\approx p$ and, thus, they cancel. Therefore, $U$ vanishes approximately for large $p$ (notice that $U_{V}$ can be neglected when $p$ becomes very large). In summary, $U$ behaves in a physically reasonable way. 
As mentioned, we have neglected the momentum dependence of $U_{S}$ and $U_{V}$. In Ref. [30] it is shown that, for low momenta, the momentum dependence of $U_{S}$ and $U_{V}$ can be described by

$$
\begin{gathered}
U_{S}(p)=U_{S}^{(0)}-U_{S}^{(1)} \frac{p^{2}}{p_{F}^{2}} \\
U_{V}(p)=U_{V}^{(0)}-U_{V}^{(1)} \frac{p^{2}}{p_{F}^{2}}
\end{gathered}
$$

with $p_{F}$ the Fermi momentum. It is found that 3, 30

$$
\frac{U_{S}^{(1)}}{U_{S}^{(0)}} \approx \frac{U_{V}^{(1)}}{U_{V}^{(0)}} \approx 0.05
$$

Expanding Eq. (13) up to terms in $p^{2}$ and using Eqs. (14)-(16), one obtains

$$
U(p) \approx U_{V}^{(0)}+U_{S}^{(0)}-0.05 \frac{U_{V}^{(0)}+U_{S}^{(0)}}{p_{F}^{2}} p^{2}+\frac{m-\tilde{m}}{2 m \tilde{m}} p^{2}
$$

The last term on the r.h.s. describes the momentum dependence of Eq. (13) for low momenta; the second but last term (which is typically a factor of five smaller) is the additional momentum dependence from $U_{S}$ and $U_{V}$ that is obviously negligible. Notice also that $U_{S}$ and $U_{V}$ are of opposite sign, but of the same order of magnitude.

The nucleon effective mass, $m^{*}$, as predicted by the DBHF calculations for nuclear matter is discussed in detail in Ref. [29]. Using the Bonn A potential, which we apply in this work, one gets $m^{*} / m=0.71$ at normal nuclear matter density $\left(\rho_{0}=0.17 \mathrm{fm}^{-3}\right)$ - a reasonable value.

Empirically, the most sophisticated investigation of the nucleon mean field (optical potential) is conducted in Dirac phenomenology [34,36. In this approach, the scalar and vector potentials are parametrized with a number of free parameters fitted to nucleon-nucleus scattering data. In order to compare our microscopic mean field obtained in DBHF calculations with the empirical one from Dirac phenomenology, we extract from the Dirac global code [36] the scalar and vector potentials in the centers of ${ }^{40} \mathrm{Ca}$ and ${ }^{208} \mathrm{~Pb}$. In Fig. 1 we compare our microscopic mean field (solid curve) at normal nuclear matter $\left(\rho_{0}=0.17 \mathrm{fm}^{-3}\right)$ with those extracted from the Dirac phenomenology. The open circles and squares correspond to 
empirical values in the center of ${ }^{208} \mathrm{~Pb}$ based on fit 1 and fit 3 of Ref. [36], respectively, while the solid triangles are the empirical values in the center of ${ }^{40} \mathrm{Ca}$ based on fit 1 of Ref. [36]. The difference between the circles and squares reflects the uncertainties in the empirical determination of the mean field, while the difference between the circles and triangles shows, to some extent, finite-size effects. In general, our microscopic result is in good agreement with the empirical one. The microscopic mean field, shows, however, a stronger momentum dependence. This may be due to the assumption of momentum independence of the scalar and vector fields in our DBHF calculation. Finite-size and surface effects, included in the Dirac phenomenology, could also play a role.

In the past, phenomenological parametrizations of momentum-dependent mean-field potentials have been constructed and used in transport models. We mention here two typical examples. One is proposed in Ref. [25] and used in BUU calculations [19], we denote it by $U^{(1)}$; the other is proposed in Ref. [24] and used in QMD calculations [21], we denote it by $U^{(2)}$. For $U^{(1)}$, one parameter set has been given [19,25] that corresponds to a soft equation of state with incompressibility $K=215 \mathrm{MeV}$; we denote this parameter set by GBG. For $U^{(2)}$, two parameter sets, corresponding to a soft and hard equation of state, have been proposed [21,24]; they are usually denoted by SMD $(K=200 \mathrm{MeV})$ and HMD $(K=380$ $\mathrm{MeV})$, respectively. In static nuclear matter, $U^{(1)}$ and $U^{(2)}$ are given by

$$
\begin{gathered}
U^{(1)}(\rho, p)=\alpha \rho+\beta \rho^{\gamma}+\delta\left(0.7965+\frac{1}{1+\mathbf{p}^{2} / \Lambda^{2}}\right) \rho \\
U^{(2)}(\rho, p)=\alpha \rho+\beta \rho^{\gamma}+\delta \ln ^{2}\left(\varepsilon \mathbf{p}^{2}+1\right) \rho^{\sigma}
\end{gathered}
$$

Parameter sets for $U^{(1)}(\mathrm{GBG})$ and $U^{(2)}$ (SMD, HMD) are listed in Table 1.

In Fig. 2, we show the density dependence of our microscopic (Eq. (13)) and of phenomenological (Eqs. (18) and (19)) mean fields. At low densities, the four mean fields shown are very close to each other. At high densities, there are significant differences: the HMD mean field is much stiffer than the others; the microscopic mean field is more close to the soft (SMD and GBG) phenomenological mean fields. There is also an important difference between the microscopic mean field obtained in our (relativistic) DBHF calculation and the 
one from non-relativistic BHF: the former is stiffer than the latter (see Fig. 4 of Ref. [15] and Fig. 3 of Ref. [17) due to repulsive relativistic effects.

In Fig. 3, we show the momentum dependence of the microscopic and phenomenological mean fields. Both microscopic and phenomenological mean fields increase with increasing momenta. For the momenta shown, the microscopic mean field has a stronger momentum dependence than the phenomenological ones. Such a strong momentum dependence is also observed in the microscopic mean field from non-relativistic BHF calculations [17]. Note, however, that for very large momenta (which are not shown in Fig. 3) the situation is reversed: the momentum-dependence disappears from our microscopic mean field (as discussed above), while the phenomenological parametrization continues to grow.

For convenience in applications, we have parametrized our microscopically derived mean field using exactly the same ansatz as for $U^{(2)}$, Eq. (19), namely,

$$
U(\rho, p)=\alpha \rho+\beta \rho^{\gamma}+\delta \ln ^{2}\left(\varepsilon \mathbf{p}^{2}+1\right) \rho^{\sigma}
$$

The parameters are listed in Table 1, row 'DBHF'. The quality of the fit can be seen in Fig. 4 where solid curves are obtained from Eq. (20) while the circles correspond to the exact calculation using Eq. (13). Both density dependence (upper part of Fig. 4) and momentum dependence (lower part of Fig. 4) are reproduced well by the parametrization Eq. (20), for the densities and momenta shown. Note, however, that the parametrization Eq. (20) is bad for large $p(p>>m)$ since it continues to grow with increasing $p$, while the exact mean field, Eq. (13), becomes independent of $p$ for large momenta. These very large momenta may, however, not be important in nucleus-nucleus collisions at intermediate energies.

The incorporation of the microscopic mean field and the in-medium NN cross sections in transport-model calculations is straightforward. The propagation of nucleons is determined by Hamilton's equations of motion 19,21

$$
\begin{gathered}
\frac{d \mathbf{p}}{d t}=-\nabla_{\mathbf{r}} U \\
\frac{d \mathbf{r}}{d t}=\frac{\mathbf{p}}{\left(\mathbf{p}^{2}+m^{2}\right)^{1 / 2}}+\nabla_{\mathbf{p}} U
\end{gathered}
$$


where the position dependence of the mean field enters through the position dependence of the density via the local-density approximation. Moreover, using microscopic in-medium NN cross sections [13], the in-medium collisions can be treated in the same way as in usual transport models. The self-consistent investigation of nucleus-nucleus collisions based upon realisitic NN interactions will be reported elsewhere.

In summary, we have derived a microscopic momentum dependent mean field using the DBHF approach for nuclear matter and the Bonn potential. The momentum dependence of our mean field comes out very close to that obtained in Dirac phenomenology. Qualitatively, our microscopic mean field shows similar density and momentum dependence as some of the phenomenological parametrizations, which are often used in BUU and QMD calculations; quantitatively, however, there are important differences, especially at high densities and momenta. For practical purposes, we have parametrized our microscopic mean field in terms of a simple analytic form which accurately reproduces our exact results; this can easily be applied in transport-model calculations.

Acknowledgement. This work is supported in part by the U.S. National Science Foundation under Grant No. PHY-9211607 and by the Idaho State Board of Education. 


\section{REFERENCES}

[1] R. Machleidt, K. Holinde and Ch. Elster, Phys. Rep. 149, 1 (1987)

[2] R. Machleidt, Adv. Nucl. Phys. 19, 189 (1989)

[3] M. R. Anastasio, L. S. Celenza, W. S. Pong and C. M. Shakin, Phys. Rep. 100 (1983) 327; L. S. Celenza and C. M. Shakin, Relativistic Nuclear Physics: Theories of Structure and Scattering, Lecture Notes in Physics, Vol. 2 (World Scientific, Singapore, 1986).

[4] B. ter Haar and R. Malfliet, Phys. Rep. 149 (1987) 207

[5] R. Brockmann and R. Machleidt, Phys. Lett. 149B, 283 (1984).

[6] R. Brockmann and R. Machleidt, Phys. Rev. C 42, 1965 (1990).

[7] Y. Miyama, Phys. Lett. 215B (1988) 602

[8] H. Müther, R. Machleidt and R. Brockmann, Phys. Rev. C 42, 1981 (1990)

[9] R. Brockmann and H. Toki, Phys. Rev. Lett. 68 (1992) 340

[10] R. Fritz, H. Müther, and R. Machleidt, Phys. Rev. Lett, 71, 46 (1993).

[11] G. Q. Li, R. Machleidt, R. Fritz, H. Müther, and Y. Z. Zhuo, Phys. Rev. C, submitted.

[12] S. Gmuca, Nucl. Phys. A547 (1992) 447

[13] G. Q. Li and R. Machleidt, Phys. Rev. C, submitted

[14] A. Bohnet, N. Ohtsuka, J. Aichelin, R. Linden and A. Faessler, Nucl. Phys. A494, 349 (1989)

[15] J. Jaenicke, J. Aichelin, N. Ohtsuka, R. Linden and A. Faessler, Nucl. Phys. A536, 201 (1992)

[16] G. Q. Li, Y. Lotfy, S. W. Huang and A. Faessler, J. of Phys. G18, 291 (1992)

[17] Dao T. Khoa, N. Ohtsuka, M. A. Matin, A. Faessler, S. W. Huang, E. Lehmann and R. 
K. Puri, Nucl. Phys. A548, 102 (1992)

[18] R. V. Reid, Ann. Phys. (N.Y.) 50, 411 (1968).

[19] G. F. Bertsch and S. Das Gupta, Phys. Rep. 160, 189 (1988)

[20] B. D. Day, Phys. Rev. Lett. 47, 226 (1981); Comments Nucl. Part. Phys. 11, 115 (1983).

[21] J. Aichelin, Phys. Rep. 202, 233 (1991)

[22] J. Cugnon, T. Mizutani and J. Vandermeulen, Nucl. Phys. A352, 505 (1981)

[23] M. Jaminon and C. Mahaux, Phys. Rev. C40, 354 (1989)

[24] J. Aichelin, A. Rosenhauer, G. Peilert, H. Stöcker and W. Greiner, Phys. Rev. Lett. 58, $1926(1987)$

[25] C. Gale, G. F. Bertsch and S. Das Gupta, Phys. Rev. C35, 1666 (1987)

[26] G. Q. Li, S. W. Huang, T. Maruyama, D. T. Khoa, Y. Lotfy and A. Faessler, Nucl. Phys. A537, 645 (1992)

[27] In this paper, we apply the so-called Bonn-A potential which is defined in Table A.2 of Ref. [2].

[28] R. H. Thompson, Phys. Rev. D 1 (1970) 710

[29] G. Q. Li, R. Machleidt and R. Brockmann, Phys. Rev. C45, 2782 (1992)

[30] R. Machleidt, in Relativistic Dynamics and Quark-Nuclear Physics, M. B. Johnson and A. Picklesimer, eds. (Wiley, New York, 1986) p. 71.

[31] B. D. Serot and J. D. Walecka, Adv. Nucl. Phys. 16, 1 (1986)

[32] K. Weber, B. Bltttel, W. Cassing, H.-C. Dönges, V. Koch, A. Lang and U. Mosel, Nucl. Phys. A539, 713 (1992)

[33] T. Maruyama, B. Blättel, W. Cassing, A. Lang, U. Mosel and K. Weber, Phys. Lett. B 
297, $228(1992)$

[34] B. C. Clark, in Relativistic Dynamics and Quark-Nuclear Structure, edited by M. B. Johnson and A. Picklesimer (Wiley, New York, 1986)

[35] D. P. Murdock and C. J. Horowitz, Phys. Rev. C 35, 1442 (1987)

[36] E. D. Cooper, S. Hama, B. C. Clark and R. L. Mercer, Phys. Rev. C 47, 297 (1993); S. Hama, private communications 
Table 1: Parameters of phenomenological and microscopic mean fields. The parameter set 'DBHF' is the parametrization of our microscopic mean field, Eq. (13), in terms of the ansatz Eq. (20). GBG uses Eq. (18), while SMD and HMD use Eq. (19).

\begin{tabular}{|c|c|c|c|c|c|c|c|}
\hline & $\alpha\left(\mathrm{MeV} \cdot \mathrm{fm}^{3}\right)$ & $\left(\mathrm{MeV} \cdot \mathrm{fm}^{3 \gamma}\right)$ & $\gamma$ & $\delta\left(\mathrm{MeV} \cdot \mathrm{fm}^{3 \sigma}\right)$ & $\varepsilon\left(\mathrm{MeV}^{-2}\right)$ & $\sigma$ & $\Lambda(\mathrm{MeV})$ \\
\hline GBG & -888.96 & 1687.53 & $7 / 6$ & -460.12 & - & - & 400 \\
\hline SMD & -2294.12 & 2412.34 & 1.14 & 9.24 & $5.0 \times 10^{-4}$ & 1.0 & - \\
\hline HMD & -764.71 & 2394.49 & 2.09 & 9.24 & $5.0 \times 10^{-4}$ & 1.0 & - \\
\hline DBHF & -1129.51 & 1501.31 & 1.48 & 35.07 & $4.3 \times 10^{-5}$ & 0.7 & - \\
\hline
\end{tabular}




\section{FIGURES}

FIG. 1. Momentum dependence of empirical and microscopically derived mean field potentials. The microscopic mean field is calculated for nuclear matter with $\rho=0.17 \mathrm{fm}^{-3}$. The empirical

mean fields are extracted from Dirac phenomenology at the centers of ${ }^{40} \mathrm{Ca}$ and ${ }^{208} \mathrm{~Pb}$, the open circles and squares correspond to ${ }^{208} \mathrm{~Pb}$ based on fit 1 and fit 3 of Ref. [36], while the solid triangles correspond to ${ }^{40} \mathrm{Ca}$ based on fit 1.

FIG. 2. Density dependence of microscopic and phenomenological mean fields for several nucleon momenta. The solid curve represents our microscopic mean field as obtained from DBHF. The dashed and dash-dotted lines are phenomenological parametrizations proposed in Ref. [24]. The dotted line is from Ref. [19,25].

FIG. 3. Momentum dependence of microscopic and phenomenological mean fields for several densities. Notation as in Fig. 2.

FIG. 4. Comparison of the parametrization Eq. (20) (solid curves) with the exact results (dots) for our microscopic mean field, Eq. (13). The upper part shows the density dependence, and the lower part the momentum dependence. 


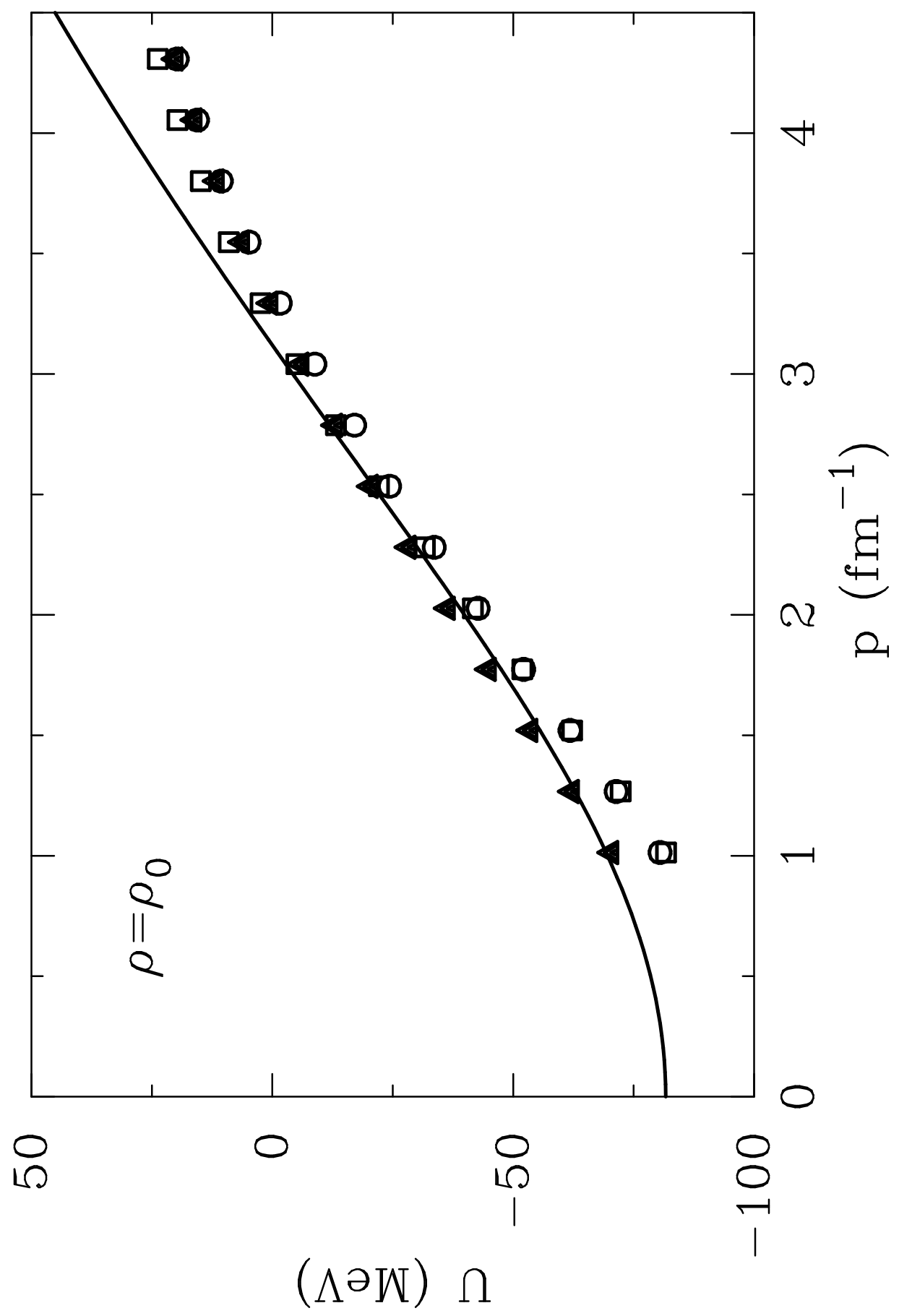



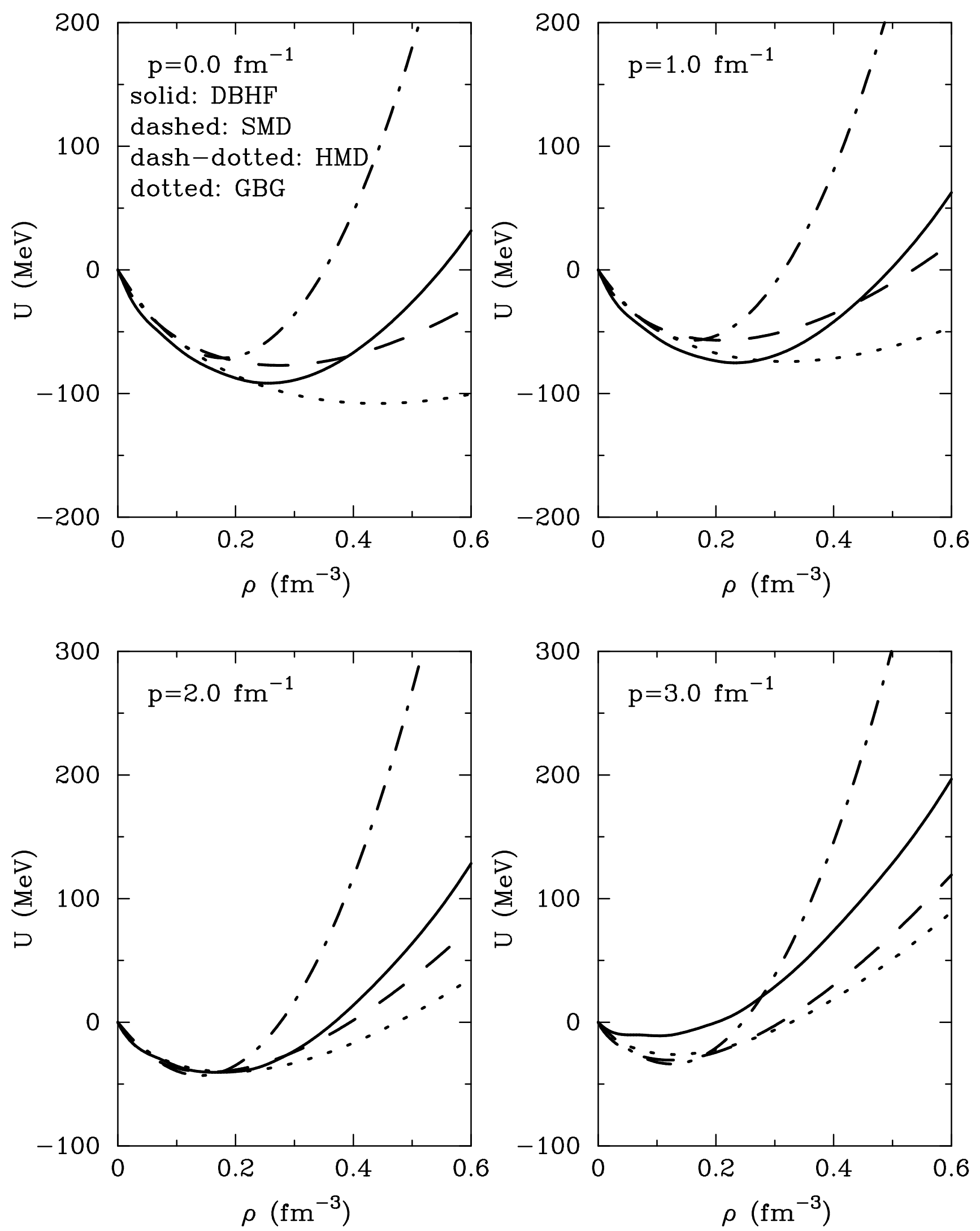

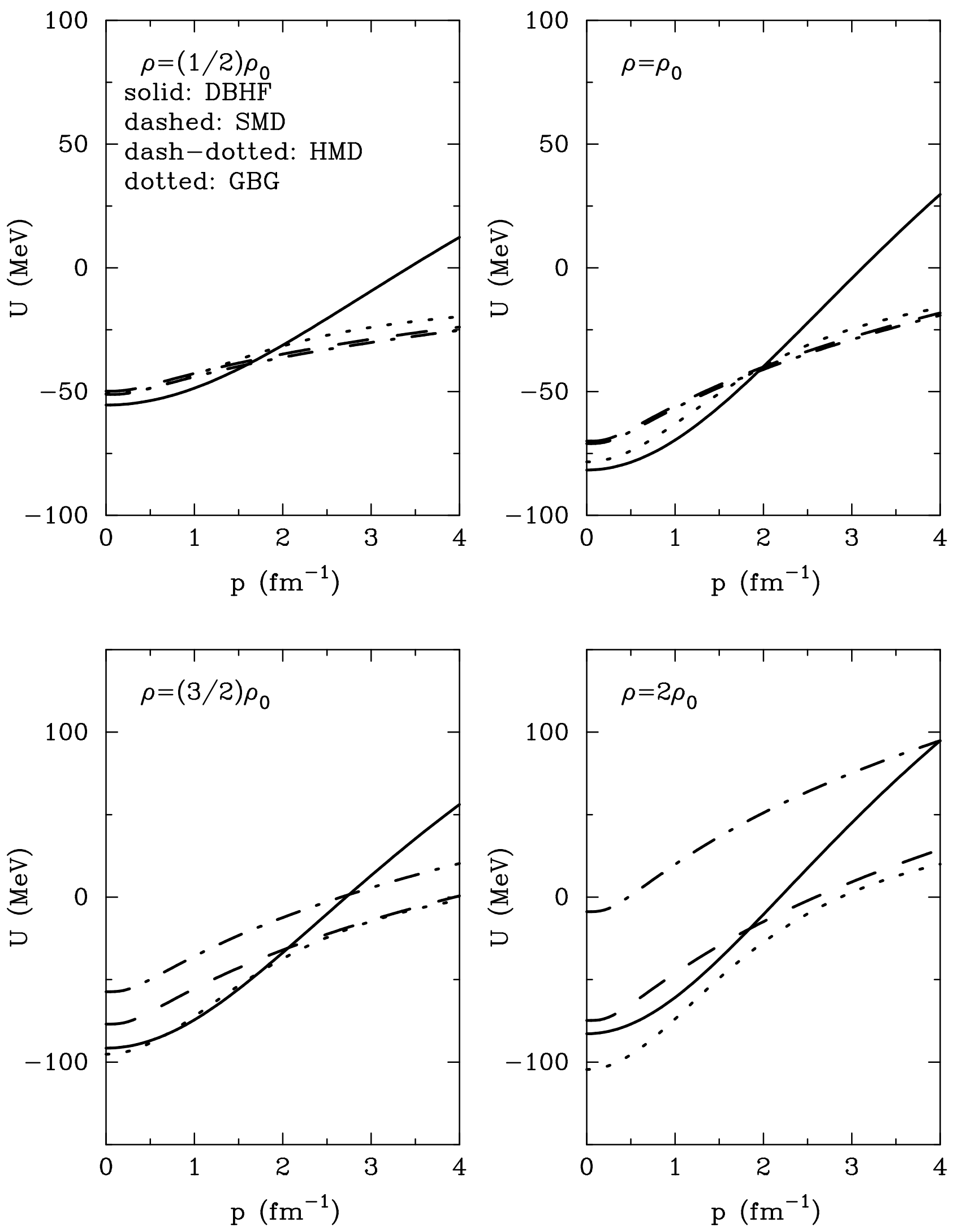

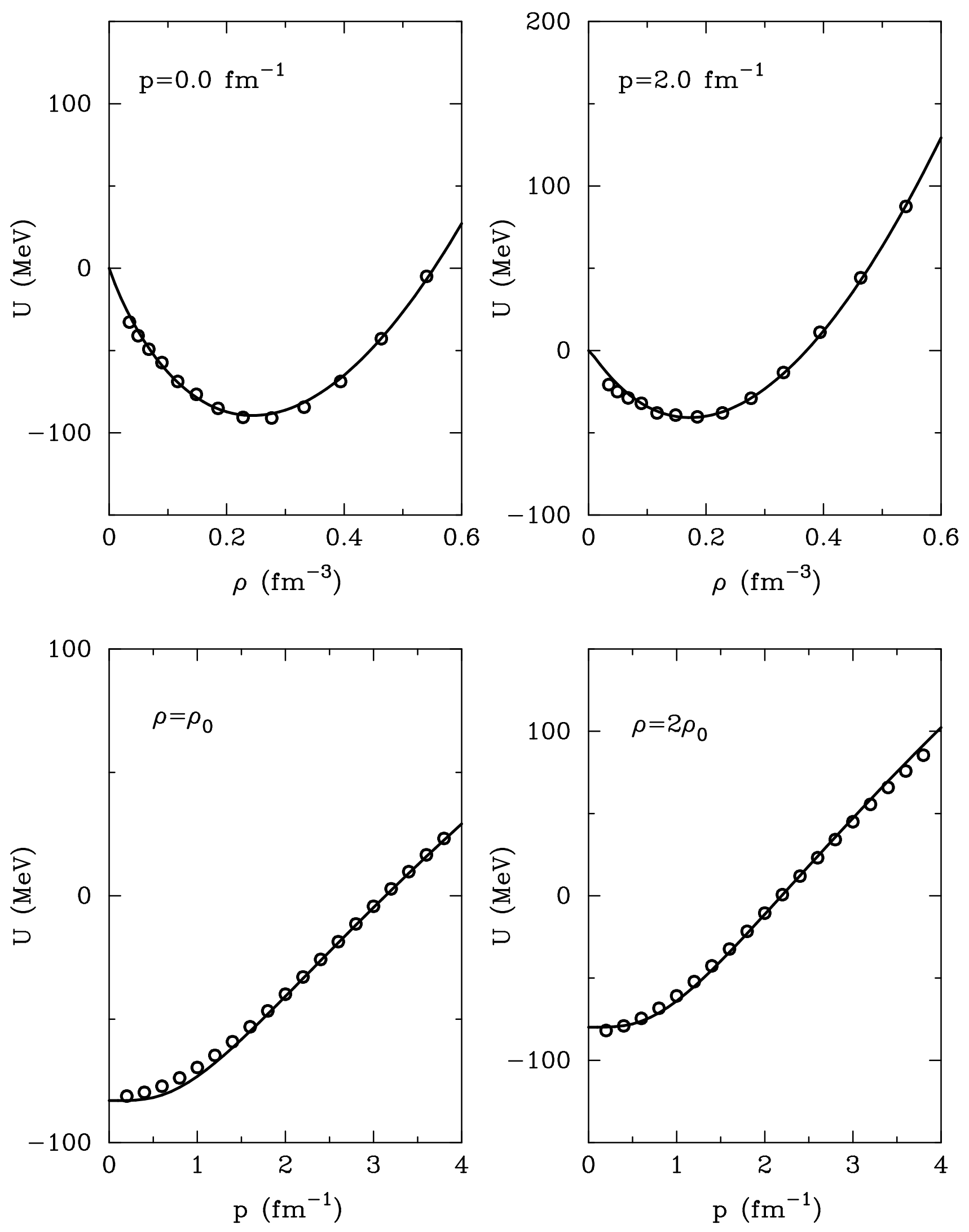